\title{
Tailoring Magnetic Behavior of CoFeMnNiX (X = Al, Cr, Ga, and Sn) High
}

\section{Entropy Alloys by Metal Doping}

Tingting Zuo ${ }^{1,2}$, Michael C. Gao ${ }^{3,4}$, Lizhi Ouyang ${ }^{5}$, Xiao Yang ${ }^{1,6}$, Yongqiang Cheng ${ }^{7}$, Rui Feng ${ }^{2}$, Shuying Chen ${ }^{2}$, Peter K. Liaw ${ }^{2}$, Jeffrey A. Hawk ${ }^{3}$, and Yong Zhang ${ }^{1}$

1. State Key Laboratory for Advanced Metals and Materials, University of Science and Technology Beijing, Beijing 100083, China

2. Department of Materials Science and Engineering, The University of Tennessee, Knoxville, TN 37996, USA

3. National Energy Technology Laboratory, 1450 Queen Ave SW, Albany, OR 97321, USA

4. AECOM, P.O. Box 1959, Albany, Albany, OR 97321, USA

5. Tennessee State University, Department of Physics and Mathematics, Nashville, TN 37209, USA

6. State Key Laboratory of Advanced Metallurgy, University of Science and Technology Beijing, Beijing 100083, China.

7. Chemical and Engineering Materials Division, Oak Ridge National Laboratory, Oak Ridge, TN 37831, USA

\begin{abstract}
Magnetic materials with excellent performances are desired for functional applications.

Based on the high-entropy effect, a system of CoFeMnNiX (X $=\mathrm{Al}, \mathrm{Cr}, \mathrm{Ga}$, and $\mathrm{Sn})$ magnetic alloys are designed and investigated. The dramatic change in phase structures

${ }^{*}$ Corresponding author: M. C. Gao. ${ }^{*}$ Corresponding author: P. K. Liaw.

${ }^{*}$ Corresponding author: Y. Zhang.

Phone: $+1-541-967-5869$ Phone: +1-865- 974-6356 Phone: +86-10-62333073

1

E-mail:michael.gao@netl.doe.gov.

E-mail: pliaw@utk.edu.

E-mail: drzhangy@ustb.edu.cn.
\end{abstract}


from face-centered-cubic (FCC) to ordered body-centered-cubic (BCC) phases, caused by adding $\mathrm{Al}, \mathrm{Ga}$, and $\mathrm{Sn}$ in $\mathrm{CoFeMnNiX}$ alloys, originates from the potent short-range chemical order in the liquid state predicted by ab initio molecular dynamics (AIMD) simulations. This phase transition leads to the significant enhancement of the saturation magnetization $\left(\mathrm{M}_{\mathrm{s}}\right)$, e.g., the CoFeMnNiAl alloy has $\mathrm{M}_{\mathrm{s}}$ of $147.86 \mathrm{Am}^{2} / \mathrm{kg}$. First-principles density functional theory (DFT) calculations on the electronic and magnetic structures reveal that the anti-ferromagnetism of $\mathrm{Mn}$ atoms in $\mathrm{CoFeMnNi}$ is suppressed especially in the CoFeMnNiAl HEA because Al changes the Fermi level and itinerant electron-spin coupling that lead to ferromagnetism.

Keywords: high entropy alloy, phase formation, magnetic behavior, AIMD simulation, DFT calculation

\section{Introduction}

The recent emergence of high-entropy alloys (HEAs), which are defined as alloys containing five or more principal elements in equal or near-equal atomic percent (at.\%), has attracted attentions by the potential breakthrough in the classical alloy design with the promise of excellent properties [1-3]. Traditionally, the search for new alloy compositions focuses on systems based on one main element. The new way of designing the alloy composition utilizing the HEA concept, which can lead to the high entropy of mixing, may suppress the formation of intermetallic compounds, and thus promote the formation of the solid-solution phase(s) in simple crystal structures, such as face-centered cubic (FCC) [3, 4], body-centered cubic (BCC) [3, 4], or hexagonal closed-pack (HCP) [3-5] lattices. Owing to their unique concentrated solid-solution 
structures, HEAs frequently possess excellent properties, such as high strength [6], high fracture toughness [7, 8], high fatigue resistance [9], good room-temperature ductility [10], good wear resistance [11], high thermal stability [12], and high electrical resistivity [13]. These outstanding properties are directly related to the so-called four "core effects" in HEAs: high entropy of mixing, sluggish diffusion, severe lattice distortion, and cocktail effects [1].

In addition to mechanical behavior, the magnetic performance of HEAs attracts great attention. Previous studies show that the high saturation magnetization $\left(\mathrm{M}_{\mathrm{s}}\right)$, low coercivity $\left(\mathrm{H}_{\mathrm{c}}\right)$, and optimal ductility can be obtained for carefully-designed alloy compositions, such as $\mathrm{FeCoNiAl}_{0.2} \mathrm{Si}_{0.2}[13,14]$. The magnetic properties of the alloys can be sensitive to the base alloy, the addition of alloying elements, and the resulting phase crystal structures $[15,16]$. For example, the addition of Si to the CoFeNi alloy can reduce the $M_{s}$ value more significantly [16] than the addition of $A 1$, while the $\mathrm{Fe}_{2} \mathrm{CoNi}$ alloy exhibits a higher $\mathrm{M}_{\mathrm{s}}$ value than $\mathrm{CoFeNi}$. This trend seems intuitive, since the non-magnetic components ( $\mathrm{Al}$ and $\mathrm{Si}$ ) are nearly non-polarized, while the ferromagnetic Fe atoms contribute significantly to the magnetic moment of the alloy. Contrary to the case of the Al/Si additions to the CoFeNi alloy, Lucas et al. [15] found that the addition of $\mathrm{Al}$ or $\mathrm{Pd}$ can turn the paramagnetism of the $\mathrm{FeCoCrNi}$ alloy at room temperature to the ferromagnetism of the $\mathrm{FeCoCrNiAl}_{2}$ alloy with the $\mathrm{M}_{\mathrm{s}}$ of $\sim 13$ $\mathrm{Am}^{2} / \mathrm{kg}$ and the ferromagnetism of the FeCoCrNiPd alloy with the $\mathrm{M}_{\mathrm{s}}$ of $33 \mathrm{Am}^{2} / \mathrm{kg}$, respectively.

More interestingly, most shape-memory Heusler alloys, such as $\mathrm{Ni}_{2} \mathrm{MnGa}$ [17], 
$\mathrm{Ni}_{2} \mathrm{MnAl}$ [18], and $\mathrm{Ni}_{2} \mathrm{MnSn}$ [19] alloys, exhibit ferromagnetism, which mostly originates from the $\mathrm{Mn}$ atoms in such a specific chemical environment further imposed by the highly-ordered lattice. The magnetic moments of the Ni atoms in these alloys are very small, and those of Al, Sn, or Ga atoms are negligible [17].

Since the Heusler alloys possess highly-ordered crystal structures in which different elements occupy specific lattice sites, the magnetic performance is closely related to the element types and their occupancies in the structure. One main objective of the present study is to investigate the magnetic behavior when mixing those elements that constitute traditional magnetic shape-memory Heusler alloys in light of the concept of HEAs. The starting base alloy is the ferromagnetic CoFeNi soft magnetic alloy. Manganese, which exhibits very weak anti-ferromagnetism at its ground state, is then added. Subsequently, the quinary $\mathrm{CoFeMnNiX}(\mathrm{X}=\mathrm{Al}, \mathrm{Ga}$, and $\mathrm{Sn})$ alloys are designed and studied. The microstructure of $\mathrm{CoFeMnNiCr}$ has been extensively studied in the literature $[7,20,21]$, but for the comparison purpose, the arc-melted $\mathrm{CoFeMnNiCr}$ is also included in the present study. Although this investigation focuses on equimolar compositions, the results and findings serve as the useful input when designing off-equimolar alloys to optimize magnetic and mechanical properties. In order to assist in fundamentally understanding the experimental observation, first-principles density-functional-theory (DFT) calculations are performed to reveal the atomic, electronic, and magnetic structures for selected single-phase alloys at zero temperature. Their atomic structures in the liquid are simulated, using the ab initio molecular dynamics (AIMD) simulations to help analyze phase formation during solidification. 


\section{Methods}

\subsection{Alloy-ingot preparation}

Alloy ingots with the nominal compositions of CoFeMnNi, CoFeMnNiAl, CoFeMnNiCr, CoFeMnNiGa, and CoFeMnNiSn were prepared by arc-melting pure elements with purity higher than 99.5 weight percent (wt. \%) under a Ti-gettered high-purity argon atmosphere on a water-cooled $\mathrm{Cu}$ hearth. The alloys were remelted five times and flipped each time in order to improve the chemical homogeneity. The prepared alloy buttons were cut into appropriate forms for investigating their microstructures, compressive mechanical behavior, and magnetic properties.

\subsection{Structural characterization and performance experiments}

Crystal structures were identified, using a (Rigaku) D/MAX-RB X-ray diffractometer. The back-scattered electron (BSE) images of cross-sections of the ingots were examined by a ZEISS SUPRA 55 field-emission-scanning-electron-microscope (SEM) with the energy-dispersive spectrometry (EDS). The transmission-electron-microscopy (TEM) investigations were conducted in an FEI Tecnai G2 F30 Super Twin operated at $200 \mathrm{kV}$. The magnetization curves were obtained by an LDJ 9600 vibrating sample magnetometer (VSM) at room temperature.

\subsection{Computational methodologies:}

\section{(1) Ab initio molecular dynamics (AIMD) simulations in the liquid state}

The AIMD simulations were performed, using the plane-wave pseudo-potential software, the Vienna Ab Initio Simulation Package (VASP) [22, 23] in a canonical ensemble, i.e., a constant mole, volume, and temperature. Newton's equations of motion 
were integrated, using the Verlet algorithm [24] with a time step of 1 fs, and the atomic-configuration relaxation and temperature were controlled by a Nose thermostat [25]. Projector augmented-wave (PAW) potentials [26] and the revised Perdew-Burke-Ernzerhof (PBE) [27] gradient approximation to the exchange-correlation functional were used. Cubic supercells of 200 atoms were built for all compositions, and the liquid densities were determined by adjusting the cell volume so that the pressure was zero in equilibrium at $\mathrm{T}=1,600{ }^{\circ} \mathrm{C}$. The simulations were performed at the $\Gamma$ point only. The electronic-energy-convergence criterion was set to 1 $\times 10^{-4} \mathrm{eV} /$ at. The default energy cutoff was used. Spin polarization was not considered. The total simulation time was more than 30 ps.

(2) First-principles density functional theory (DFT) calculations at zero temperature

The total energy calculations at zero temperature were carried out on the FCC-structured CoFeMnNi and CoFeMnNiCr, and BCC-structured CoFeMnNiAl HEAs using VASP. The input atomic structures of the disordered equimolar quaternary FCC, quinary FCC, and quinary $\mathrm{BCC}$ structures were constructed, using the special quasi-random structures (SQS) [28] through the Alloy Theoretic Automated Toolkit (ATAT) package developed by Axel van de Walle and co-workers [29, 30]. The 64-atom FCC, 125-atom FCC, and 125-atom BCC SQS models are detailed elsewhere [31]. The PAW potentials [26] and the PBE [32] gradient approximation for the exchange-correlation functional were used. The Brillouin-zone integrations were performed, using the Monkhorst-Pack k-point meshes [33], and the k-meshes used were 
$4 \times 4 \times 4$ and $3 \times 3 \times 3$ for the quaternary and quinary SQS models, respectively. A smearing parameter of $0.2 \mathrm{eV}$ was chosen for the Methfessel-Paxton [34] technique. The plane-wave energy cutoff is held constant at $300 \mathrm{eV}$. All structures in the non-magnetic state (i.e., without considering polarization) were optimized first, and then were re-optimized considering collinear spin polarization (i.e., using ferromagnetic and anti-ferromagnetic spin configurations as the initial input, respectively). Both lattice parameters and atomic coordinates were fully relaxed under zero pressure until the energy convergence reached $1 \mathrm{meV} /$ atom. The configuration that resulted in the lowest energy was used for data analysis.

\section{Results}

\subsection{Crystal structures}

Figure 1 shows the X-ray diffraction (XRD) profiles of the alloys in the as-cast state. The quaternary CoFeMnNi alloy possesses a simple FCC structure with a lattice constant of about $3.5985 \AA$, while the mostly-investigated CoFeMnNiCr alloy also exhibits a simple FCC structure with the elements distributing uniformly in the alloys. Although the XRD pattern for the CoFeMnNiAl alloy cannot exclusively reveals whether it is a single B2 phase or a mixture of both B2 and disordered BCC phases, the in-depth information obtained by TEM in Fig. 2 shows that a large amount of nanoparticle distributed uniformly in the matrix [Fig. 2(a)]. The dark-field TEM micrograph in Fig. 2(b), imaged using the [l $\left.\begin{array}{lll}0 & 0 & 1\end{array}\right]$ superlattice reflection, reveals an ordered BCC-structured matrix (i.e., B2 structure, bright contrast). The dark nano-scaled particles should be the disordered BCC structure. The corresponding $\left[\begin{array}{lll}1 & 0 & 0\end{array}\right]$ 
zone axis diffraction pattern in Fig. 2(c) verifies the presence of an ordered BCC structure.

The CoFeMnNiGa alloy shows the coexistence of simple FCC and BCC phases, and the volume fraction of the BCC phase is much higher than the FCC phase according to the intensity of the diffraction peaks from the XRD pattern. The in-depth information of the CoFeMnNiGa alloy detected by TEM in Fig. 2(d)-(f) shows that the dendrite-like phase has an FCC structure [Fig. 2(e)], while the matrix phase has a B2 structure [Fig. 2(f)]. By measuring the electron-diffraction spots, the lattice constants of B2 and FCC phases are $2.852 \AA$ and $3.676 \AA$, respectively. With the energy-dispersive spectroscopy (EDS) results (Table 1), the dendrites (DR) are rich in Fe and Co, while the matrix is rich in $\mathrm{Ni}, \mathrm{Mn}$, and $\mathrm{Ga}$.

The CoFeMnNiSn alloy presents a more complex microstructure: The main phase is similar to the $\mathrm{Co}_{2} \mathrm{MnSn}$ phase, which belongs to the space group of $F m 3 m$ with the L2 1 structure. A second phase with the BCC structure also appears, and the lattice constant of this phase is $2.899 \AA$. A small peak at $2 \theta \approx 43.5^{\circ}$ cannot be indexed and may indicate another phase in a very small amount. The TEM results presented in Fig. 2(g)-(i) show that the spherical precipitates with a diameter of about $0.5 \sim 1 \mu \mathrm{m}$ distributed in the matrix. By examining the electron-diffraction pattern from the particle at the [011] zone axis, the precipitate is determined to be the BCC structure. The typical diffraction pattern of the $\mathrm{L} 2{ }_{1}$ structure from the [011] zone axis also confirmed that the matrix is a $\mathrm{Co}_{2} \mathrm{MnSn}$-like phase, and its composition (Table 1) suggests that $\mathrm{Fe}$ and Ni substitute for Co and Mn. The measured lattice constant of the matrix is about 
$6.056 \AA$, which is slightly larger than the pure $\mathrm{Co}_{2} \mathrm{MnSn}$ phase $(a=5.989 \AA)$. From the EDS analysis, the precipitates are rich in $\mathrm{Fe}$ and $\mathrm{Co}$, while the matrix is rich in $\mathrm{Ni}, \mathrm{Mn}$, and Sn.

\subsection{Magnetic behavior}

The magnetic hysteresis loops of the CoFeMnNiX (X = Al, Ga, and $\mathrm{Sn})$ alloys shown in Fig. 3 resemble the typical ferromagnetic behavior of CoFeMnNiAl, CoFeMnNiGa, and CoFeMnNiSn, while the magnetic hysteresis loop of the CoFeMnNiCr alloy is similar to the paramagnetic behavior. The corresponding parameters deduced from the hysteresis loops are also listed in Fig. 3. For the CoFeMnNi alloy, its saturation magnetization $\left(\mathrm{M}_{\mathrm{s}}\right)$ and coercivity $\left(\mathrm{H}_{\mathrm{c}}\right)$ are 18.14 $\mathrm{Am}^{2} / \mathrm{kg}$ and $119 \mathrm{~A} / \mathrm{m}$, respectively. Adding $\mathrm{Al}, \mathrm{Ga}$, or $\mathrm{Sn}$ to the CoFeMnNi base alloy increases both the $\mathrm{M}_{\mathrm{s}}$ and $\mathrm{H}_{\mathrm{c}}$ values noticeably. For example, the CoFeMnNiAl alloy has $\mathrm{M}_{\mathrm{s}}$ of about $147.86 \mathrm{Am}^{2} / \mathrm{kg}$ and $\mathrm{H}_{\mathrm{c}}$ of approximately $629 \mathrm{~A} / \mathrm{m}$. CoFeMnNiGa and CoFeMnNiSn have nearly the same $\mathrm{M}_{\mathrm{s}}$ at about $80 \mathrm{Am}^{2} / \mathrm{kg}$, but very different $\mathrm{H}_{\mathrm{c}}$. The $\mathrm{H}_{\mathrm{c}}$ is $915 \mathrm{~A} / \mathrm{m}$ for CoFeMnNiGa, but reaches 3,431 A/m for CoFeMnNiSn, which is beyond the scope of soft magnetic materials (for soft magnetic materials, $\mathrm{H}_{\mathrm{c}}<1,000$ $\mathrm{A} / \mathrm{m})$. In contrast, CoFeMnNiCr doesn't saturate even when the magnetic field reaches $5.0 \times 10^{6} \mathrm{~A} / \mathrm{m}$, and its $\mathrm{M}_{\mathrm{s}}$ is only $1.39 \mathrm{Am}^{2} / \mathrm{kg}$ while having the largest $\mathrm{H}_{\mathrm{c}}$ value of $10,804 \mathrm{~A} / \mathrm{m}$.

\section{Discussions}

Magnetic materials play an important role in today's materials technologies. One of the most important characteristics desired for either soft or hard magnetic materials is 
the high saturation magnetization. The saturation magnetization in this series of alloys exhibits interesting behavior, especially when compared with the $\mathrm{CoFeNi}(\mathrm{AlSi})_{\mathrm{x}}$ and $\mathrm{CoFeNiAl}_{\mathrm{x}}$ series $[13,16]$. The CoFeNi base alloy has a very high saturation magnetization $\left(149.65 \mathrm{Am}^{2} / \mathrm{kg}\right.$ ), but it decreases to $49.9 \mathrm{Am}^{2} / \mathrm{kg}$ in $\mathrm{CoFeNi}(\mathrm{AlSi})_{0.8}$, and $101.77 \mathrm{Am}^{2} / \mathrm{kg}$ in CoFeNiAl. The resultant behavior in $\mathrm{CoFeNi}(\mathrm{AlSi})_{\mathrm{x}}$ and $\mathrm{CoFeNiAl}_{\mathrm{x}}$ has a relatively-straightforward explanation: Adding the non-magnetic elements to the ferromagnetic $\mathrm{CoFeNi}$ alloy will reduce the magnetization regardless of the phase structure of the alloying elements.

In the current $\mathrm{CoFeMnNiX}$ series, however, their magnetic properties are not as simple as $\mathrm{CoFeNi}(\mathrm{AlSi})_{\mathrm{x}}$. First of all, the starting alloy, CoFeMnNi, has a tremendously lower saturation magnetization $\left(18.14 \mathrm{Am}^{2} / \mathrm{kg}\right)$, compared to CoFeNi (149.65 $\left.\mathrm{Am}^{2} / \mathrm{kg}\right)$, albeit they have the same FCC structure. The reduction of $\mathrm{M}_{\mathrm{s}}$ due to the Mn addition is presumably because Mn favors the anti-ferromagnetic order with the other elements in this alloy. This feature is similar to the role that $\mathrm{Cr}$ plays in the case of $\mathrm{CoCrFeNi}$ [13], and is correlated with the fact that both $\mathrm{Mn}$ and $\mathrm{Cr}$ are antiferromagnetic in their ground states. Furthermore, adding $\mathrm{Cr}$ to $\mathrm{CoFeMnNi}$ dramatically reduces the saturation magnetization to $1.39 \mathrm{Am}^{2} / \mathrm{kg}$ due to the stronger magnetization cancelling effect of $\mathrm{Cr}$, compared to Mn. Consequently, this is associated with its much higher Néel temperature $\left(35^{\circ} \mathrm{C}\right.$ for $\mathrm{Cr}$ vs. $-173{ }^{\circ} \mathrm{C}$ for $\left.\mathrm{Mn}\right)$.

However, intriguing magnetic properties are observed when $\mathrm{Al}$ (or $\mathrm{Ga} / \mathrm{Sn}$ ) is introduced into the CoFeMnNi alloy. In sharp contrast to $\mathrm{CoFeNi}(\mathrm{AlSi})_{\mathrm{x}}[13,16]$, the saturation magnetization of CoFeNiMnAl is enhanced by one order of magnitude to 
147.86 $\mathrm{Am}^{2} / \mathrm{kg}$, which is comparable to that of FeCoNi. With the Ga or Sn addition, there is also a significant increase to about $80 \mathrm{Am}^{2} / \mathrm{kg}$. The apparent enhancement in $\mathrm{M}_{\mathrm{s}}$ of CoFeMnNiX alloys, compared to the base CoFeMnNi alloy, is due to the dramatic change in the structures of stable phases, which accordingly influences their unique electronic and magnetic structures. It is hypothesized that the original anti-ferromagnetic order associated with $\mathrm{Mn}$ atoms in $\mathrm{CoFeMnNi}$ is suppressed to favor ferromagnetism due to doping of $\mathrm{Al}, \mathrm{Ga}$, or $\mathrm{Sn}$.

To further illustrate the change in electronic and magnetic structures due to doping in $\mathrm{CoFeMnNi}$, the DFT calculations of the electronic structures were performed on selected compositions. Fig. 4(a) shows that the total electron density of states (DOS) for up and down spins of $\mathrm{CoFeMnNi}$ and $\mathrm{CoFeMnNiCr}$ are more symmetric than CoFeMnNiAl, while there is a significantly-higher spin-up distribution than the spin-down distribution in CoFeMnNiAl. The $d$-orbital DOS of transition metals plays a crucial role in their physical properties. Thus, the partial DOS (PDOS) of $d$-orbitals of Co, Fe, Mn, Ni, Al and $\mathrm{Cr}$ are presented in Fig. 4(b)-(f), respectively. The most notable change upon the addition of $\mathrm{Al}$ is the shift of a $\mathrm{Mn}$ majority spin peak from above the Fermi level to below the Fermi level, caused by a switch from the anti-ferromagnetic order to ferromagnetic order, which leads to a significant increase of the magnetization of CoFeMnNiAl [Fig. 4(d)]. Part of the Fe majority spin states above the Fermi level also shifts below the Fermi level, further enhancing its magnetization [Fig. 4(c)]. In general, the non-magnetic $\mathrm{Al}$ reduces the $d$-band peak width, compared to CoFeMnNi and CoFeMnNiCr. As shown in Fig. 4(b), the addition of Cr leads to Co (down-spin 
PDOS) going from the high spin state to low spin state by shifting the minority peak above the Fermi level to below the Fermi level. The impact on PDOS of Ni due to the $\mathrm{Al}$ or $\mathrm{Cr}$ addition is small [Fig. 4(e)]. The PDOS of $\mathrm{Al}$ and $\mathrm{Cr}$ atoms [Fig. 4(f)] for spin-up and spin-down distributions are quite symmetric, suggesting that their direct contributions to the magnetization of the alloys are negligible, although their additions alter the neighboring environments of $\mathrm{Mn}$ and other elements. In particular, CoFeMnNiAl prefers the BCC over FCC structure.

Since both BCC and FCC structures have a point group symmetry, $\mathrm{O}_{\mathrm{h}}$, the crystal field is supposedly to split the $d$-orbitals into two groups: $\mathrm{t}_{2 \mathrm{~g}}\left(\mathrm{~d}_{\mathrm{xy}}, \mathrm{d}_{\mathrm{yz}}, \mathrm{d}_{\mathrm{xz}}\right)$ and $\mathrm{e}_{\mathrm{g}}\left(\mathrm{d}_{\mathrm{z} 2}\right.$, $d_{x 2-y 2}$. The orbital-decomposed PDOS of Mn in terms of $t_{2 g}$ and $e_{g}$ orbitals is shown in Fig. 4(g). The Jahn-Teller effect [35] and the substitutional disorders (different atomic configurations around $\mathrm{Mn}$ sites) lead to further splitting of $\mathrm{t}_{2 \mathrm{~g}}$ and $\mathrm{e}_{\mathrm{g}}$ bands. While the addition of $\mathrm{Cr}$ to $\mathrm{CoFeMnNii}$ causes little change to the $\mathrm{Mn} d$-band, the addition of $\mathrm{Al}$ significantly reduces the $\mathrm{Mn} d$-band width and increases the exchange splitting. The most striking change from the double $\mathrm{e}_{\mathrm{g}}$ bands to a single band is due to the change from the anti-ferromagnetic order to ferromagnetic order for Mn sites, as revealed by the site-decomposed PDOS analysis.

The predicted local magnetic moments of individual atoms in FCC CoFeMnNi, FCC CoFeMnNiCr, and $\mathrm{BCC}$ CoFeMnNiAl at zero temperature are shown in Fig. 5. For all alloys, Fe exhibits a higher magnetic moment than Co, while Ni presents a near-zero moment. $\mathrm{Co}, \mathrm{Fe}$, and $\mathrm{Ni}$ behave ferromagnetic-like in $\mathrm{CoFeMnNi}$, while $\mathrm{Mn}$ behaves antiferromagnetic-like. The addition of $\mathrm{Cr}$ to $\mathrm{CoFeMnNi}$ overall lowers the 
magnitude of the magnetic moment, and causes larger scattering in the moment of the atoms, suggesting the sensitivity of the moment to neighboring atoms due to the $\mathrm{Cr}$ addition. On the other hand, the addition of $\mathrm{Al}$ to $\mathrm{CoFeMnNi}$ reduces the number of down-spins for $\mathrm{Mn}$, and thus, causes the alloy to behave ferromagnetic-like. The calculated average magnetic moments are $0.89,0.39$, and $1.23 \mu_{\mathrm{B}} /$ atom $\left(\mu_{\mathrm{B}}=\mathrm{Bohr}\right.$ magneton) for $\mathrm{CoFeMnNi}, \mathrm{CoFeMnNiCr}$, and $\mathrm{CoFeMnNiAl}$, respectively, while the corresponding experimental values are $0.19,0.014$, and $1.35 \mu_{\mathrm{B}}$ /atom.

It is expected that the predicted magnetic moment of the alloy may differ from the experimental measurements. The main reasons are: (1) the DFT calculations are conducted at zero temperature with no consideration for temperature effects, which means that the magnetic moment can reach the theoretically maximum condition; (2) the DFT calculations are performed on equimolar alloys, using the disordered SQS models, while the true alloy compositions may deviate from the nominal ones with the true atomic structure being more complex. For example, the presence of B2 and disordered $\mathrm{BCC}$ phases were observed in the as-cast CoFeMnNiAl alloy, and it impacts both the phase composition and the surrounding environment of different atoms in the lattices, which leads to various electronic/magnetic interactions; and (3) chemical and structural heterogeneities in the as-cast samples introduce the additional complexity to the magnetic behavior observed experimentally.

Achieving a single-phase microstructure was not the objective of the present study due to the apparently large differences in the atomic size and chemical nature between $\mathrm{Al} / \mathrm{Ga} / \mathrm{Sn}$ and $\mathrm{Co} / \mathrm{Fe} / \mathrm{Mn} / \mathrm{Ni}$ elements. However, studying their atomic structures in the 
liquid will, in turn, aid in understanding better the phase formation during solidification. In fact, the formation of ordered phases in CoFeMnNiX alloys is not a surprise, as the AIMD simulations (see Fig. 6) reveal the formation of significant short-range order in these alloys, which can be reflected by the first-nearest-neighbor (FNN) pairs in the partial pair distribution functions (PDFs).

For example, the noticeable preference in NiAl, NiGa, and NiSn FNN pairs are observed due to the additions of $\mathrm{Al}, \mathrm{Ga}$, and $\mathrm{Sn}$ to the base $\mathrm{CoFeMnNi}$ alloy, respectively, suggesting the local short-range order of $\mathrm{NiAl}, \mathrm{NiGa}$, and $\mathrm{NiSn}$. These predicted structural features on the atomic level in the liquid are consistent with the experimental observation of the formation of the NiAl-rich B2 phase in the CoFeMnNiAl alloy, the Ni-Mn-Ga rich B2-structured matrix in the CoFeMnNiGa alloy, and the $\mathrm{Ni}_{2} \mathrm{MnSn}$-type $\mathrm{L} 2{ }_{1}$-structured compound in the CoFeMnNiSn alloy. Similar phenomena like an isomorphous solid solution of the $\mathrm{B} 2(\mathrm{Co}, \mathrm{Ni}) \mathrm{Al}$ phase at $1,300{ }^{\circ} \mathrm{C}$ in the ternary Al-Co-Ni and B2 $(\mathrm{Co}, \mathrm{Ni}) \mathrm{Ga}$ phase at $1,000{ }^{\circ} \mathrm{C}$ in the ternary $\mathrm{Co}-\mathrm{Ga}-\mathrm{Ni}$ were reported by Kainuma et al. [36] and by Ducher et al. [37], respectively. The congruent formation of the $\mathrm{L} 2{ }_{1} \mathrm{Ni}_{2} \mathrm{MnSn}$ compound at $1,140{ }^{\circ} \mathrm{C}$ in the ternary Mn-Ni-Sn with a wide compositional range at lower temperatures was reported by Laudise et al. [38]. Therefore, it is common to form ordered phases when alloying $\mathrm{Ni}, \mathrm{Mn}$, and $\mathrm{Al} / \mathrm{Sn} / \mathrm{Ga}$ together.

The close relationship between the short-range order and segregation in the liquid and phase formation during solidification demonstrates the important role of the liquid structure in the development of the solid-state microstructure of HEAs. For example, 
the addition of an element, which causes significant short-range order or segregation in the liquid, will promote the formation of the intermetallic compound or phase separation during solidification. This trend is basically consistent with the prior AIMD simulations carried out on the single-phase solid-solution HEAs (e.g., HfNbTaTiZr [3, 39], HfNbTaTiVZr [40], GdDyLuTbY [5], CoOsReRu [5], and MoPdRhRu [5]), multi-phase alloy $\left(\mathrm{Al}_{1.3} \mathrm{CoCrCuFeNi}\right)[3,39,41]$, and high-entropy bulk metallic glass-forming alloys (i.e., CuNiPPdPt [3, 39] and AlErDyNiTb, CuHfNiTiZr, and BeCuNiTiZr [42]). These simulations point to a conclusion $[3,5,39-42]$ that a liquid structure that lacks strong elemental segregation or potent short-range order will promote the formation of disordered solid solutions during solidification.

\section{Conclusions}

To summarize, the structural and magnetic behaviors of this new class of HEAs are novel and attractive. The magnetic properties of these alloys have been experimentally and theoretically studied and compared with the observed atomic-level and micro-scale structures. Both the DFT and AIMD simulations are used to help understand the intrinsic characteristics of the HEAs, which can provide guidelines for designing HEAs. The present research offers a pathway to the fundamental study and development of structural and functional HEAs. From this investigation, we can obtain the following conclusions:

(1) Ordered phases form by adding $\mathrm{Al} / \mathrm{Ga} / \mathrm{Sn}$ to the $\mathrm{FCC}$-structured $\mathrm{CoFeMnNi}$ alloy. The CoFeMnNiAl alloy presents both $\mathrm{B} 2$ and $\mathrm{BCC}$ phases, with the BCC-structured nanoparticles distributed in the B2 matrix. The CoFeMnNiGa alloy 
possesses a B2-structured matrix and a FCC-structured second phase, while the CoFeMnNiSn alloy presents high-volume-fraction BCC-structured precipitates distributed uniformly in the $\mathrm{L} 2{ }_{1}$-structured matrix.

(2) The AIMD simulations indicate that the addition of $\mathrm{Al}, \mathrm{Ga}$, or $\mathrm{Sn}$ can cause strong short-range order in the liquid, which favors the formation of ordered phases during solidification.

(3) The CoFeMnNiX (X = Al, Ga, and Sn) alloys exhibit typically ferromagnetic behavior, with significantly increased $M_{s}$ (e.g., the $M_{s}$ reaches $147.86 \mathrm{Am}^{2} / \mathrm{kg}$ for CoFeMnNiAl), because the crystal and electronic structures of CoFeMnNi are altered by the addition of $\mathrm{Al}, \mathrm{Ga}$, or $\mathrm{Sn}$. The $\mathrm{DFT}$ calculation predicts that the anti-ferromagnetic order associated with $\mathrm{Mn}$ in the FCC CoFeMnNi is suppressed to favor ferromagnetism in the $\mathrm{BCC}$ CoFeMnNiAl. In contrast, the CoFeMnNiCr alloy behaves paramagnetic-like.

In the future work, efforts can be put on the following areas: (1) searching for potential soft magnetic HEAs based on the compositions of $\mathrm{CoFe}(\mathrm{Cr}, \mathrm{Mn})_{\mathrm{x}} \mathrm{Ni}(\mathrm{Al}, \mathrm{Ga})_{\mathrm{y}}$. The contents of $\mathrm{Cr}, \mathrm{Mn}, \mathrm{Al}$, and $\mathrm{Ga}$ can be further optimized; (2) studying the L2 ${ }_{1}$-structued $\mathrm{Ni}_{2} \mathrm{MnAl}, \mathrm{Ni}_{2} \mathrm{MnGa}$ and $\mathrm{Ni}_{2} \mathrm{MnSn}$ type HEAs, and the $\mathrm{Ni}$ or $\mathrm{Mn}$ sites can be occupied by two or more elements from the family of $\mathrm{Co}, \mathrm{Fe}, \mathrm{Mn}$, and $\mathrm{Ni}$ elements. They may include $\mathrm{Ni}_{2}(\mathrm{CoMn}) \mathrm{X}, \quad \mathrm{Ni}_{2}(\mathrm{FeMn})_{1} \mathrm{X}, \quad(\mathrm{CoNi})_{2} \mathrm{MnX}, \quad(\mathrm{FeNi})_{2} \mathrm{MnX}$, $(\mathrm{CoNi})_{2}(\mathrm{FeMn}) \mathrm{X},(\mathrm{FeNi})_{2}(\mathrm{CoMn}) \mathrm{X}, \mathrm{Ni}_{2}(\mathrm{CoFeMn}) \mathrm{X}$, and $(\mathrm{CoFeNi})_{2} \mathrm{MnX}(\mathrm{X}=\mathrm{Al}, \mathrm{Ga}$ and Sn) as well as derivatives of these compositions. First-principles DFT calculations on these proposed alloys can be carried out prior to experiments in order to accelerate 
the alloy design and lower the cost. Despite the fact that much work remains to develop the functional HEA for applications, the idea reported here offers a strong motivation to pursue the future development of HEAs.

\section{Acknowledgement}

This project was supported by the National High Technology Research and Development Program of China (No. 2009AA03Z113) and the National Science Foundation of China (Nos. 51471025 and 51210105006), 111 Project (B07003), and the Program for Changjiang Scholars and the Innovative Research Team of the University. P.K. Liaw very much appreciates the financial support from the US National Science Foundation (DMR-1611180) with D. Farkas as the contract monitor. P.K. Liaw is very grateful for the support from the US Department of Energy (DOE), Office of Fossil Energy, National Energy Technology Laboratory (NETL) (DE-FE-0008855, DE-FE-0011194, and DE-FE-0024054), and the US Army Research Office (W911NF-13-1-0438) with V. Cedro, J. Mullen, S. Markovich, R. Dunst, S. Mathaudhu, D. Stepp, and M. P. Bakas as contract monitors. M.C. Gao and J.A. Hawk acknowledge financial support by the Cross-Cutting Technologies Program at the NETL-Strategic Center for Coal, managed by Robert Romanosky (Technology Manager) and Charles Miller (Technology Monitor). The computing portion of the research was executed through the NETL's Office of Research and Development's Innovative Process Technologies (IPT) Field Work Proposal. The research performed by the AECOM Staff was conducted under the RES contract DE-FE-0004000. M.C. Gao wishes to thank Chao Jiang for sharing the SQS structures, and Mike Widom, and David Alman for 
discussions on the HEA topic. L. Ouyang thanks the financial support from NETL (DE-FE-0011549). Y.Q. Cheng is supported by the Scientific User Facilities Division, Office of Basic Energy Sciences, United States Department of Energy. Computational resources were made available through the Center of Nanophase Materials Sciences, Oak Ridge National Laboratory.

\section{References}

[1] J.W. Yeh, S.K. Chen, S.J. Lin, J.Y. Gan, T.S. Chin, T.T. Shun, C.H. Tsau, S.Y. Chang. Nanostructured high-entropy alloys with multiple principal elements: novel alloy design concepts and outcomes, Adv. Eng. Mater. 6 (2004) 299-303.

[2] B. Cantor, I.T.H. Chang, P. Knight, A.J.B. Vincent. Microstructural development in equiatomic multicomponent alloys, Mater. Sci. Eng., A 375-377 (2004) 213-218.

[3] Y. Zhang, T.T. Zuo, Z. Tang, M.C. Gao, K.A. Dahmen, P.K. Liaw, Z.P. Lu. Microstructures and properties of high-entropy alloys, Prog. Mater Sci. 61 (2014) 1-93.

[4] M.C. Gao. Chapter 11: Design of High-Entropy Alloys. in: M.C. Gao, J.W. Yeh, P.K. Liaw, Y. Zhang, (Eds.). High-Entropy Alloys: Fundamentals and Applications. Springer, Cham, Switzerland, 2016.

[5] M.C. Gao, B. Zhang, S.M. Guo, J.W. Qiao, J.A. Hawk. High-entropy alloys in hexagonal close packed structure, Metall. Mater. Trans. A 47 (2015) 3322-3332.

[6] O.N. Senkov, S.V. Senkova, C. Woodward. Effect of aluminum on the microstructure and properties of two refractory high-entropy alloys, Acta Mater 68 (2014) 214-228.

[7] B. Gludovatz, A. Hohenwarter, D. Catoor, E.H. Chang, E.P. George, R.O. Ritchie. A 
fracture-resistant high-entropy alloy for cryogenic applications, Science 345 (2014) $1153-1158$.

[8] Z. Zhang, M.M. Mao, J. Wang, B. Gludovatz, Z. Zhang, S.X. Mao, E.P. George, Q. Yu, R.O. Ritchie. Nanoscale origins of the damage tolerance of the high-entropy alloy CrMnFeCoNi, Nat Commun 6 (2015) 10143.

[9] M.A. Hemphill, T. Yuan, G.Y. Wang, J.W. Yeh, C.W. Tsai, A. Chuang, P.K. Liaw. Fatigue behavior of $\mathrm{Al}_{0.5} \mathrm{CoCrCuFeNi}$ high entropy alloys, Acta Mater 60 (2012) $5723-5734$.

[10]F. Otto, A. Dlouhý, C. Somsen, H. Bei, G. Eggeler, E.P. George. The influences of temperature and microstructure on the tensile properties of a $\mathrm{CoCrFeMnNi}$ high-entropy alloy, Acta Mater 61 (2013) 5743-5755.

[11] M.H. Chuang, M.H. Tsai, W.R. Wang, S.J. Lin, J.W. Yeh. Microstructure and wear behavior of $\mathrm{Al}_{\mathrm{x}} \mathrm{Co}_{1.5} \mathrm{CrFeNi}_{1.5}$ Tiy high-entropy alloys, Acta Mater 59 (2011) 6308-6317. [12]W.R. Wang, W.L. Wang, J.W. Yeh. Phases, microstructure and mechanical properties of $\mathrm{Al}_{\mathrm{x}} \mathrm{CoCrFeNi}$ high-entropy alloys at elevated temperatures, J. Alloys Compd. 589 (2014) 143-152.

[13]Y. Zhang, T. Zuo, Y. Cheng, P.K. Liaw. High-entropy alloys with high saturation magnetization, electrical resistivity, and malleability, Sci Rep 3 (2013) 1455.

[14]T. Zuo, X. Yang, P.K. Liaw, Y. Zhang. Influence of Bridgman solidification on microstructures and magnetic behaviors of a non-equiatomic FeCoNiAlSi high-entropy alloy, Intermetallics 67 (2015) 171-176.

[15]M.S. Lucas, L. Mauger, J.A. Munoz, Y.M. Xiao, A.O. Sheets, S.L. Semiatin, J. 
Horwath, Z. Turgut. Magnetic and vibrational properties of high-entropy alloys, J. Appl. Phys. 109 (2011) 3.

[16]T.T. Zuo, R.B. Li, X.J. Ren, Y. Zhang. Effects of Al and Si addition on the structure and properties of CoFeNi equal atomic ratio alloy, J. Magn. Magn. Mater. 371 (2014) $60-68$.

[17]P.J. Webster, K.R.A. Ziebeck, S.L. Town, M.S. Peak. Magnetic order and phase-transformation in $\mathrm{Ni}_{2} \mathrm{MnGa}$, Philos Mag B 49 (1984) 295-310.

[18]Galanakis, E. Şaşığlu. Structural-induced antiferromagnetism in Mn-based full Heusler alloys:The case of $\mathrm{Ni}_{2} \mathrm{MnAl}$, Appl. Phys. Lett. 98 (2011) 102514.

[19]H.S. Mund, A. Dashora, J. Sahariya, K.C. Bhamu, K.R. Priolkar, N. Lobo, M. Itou, Y. Sakurai, B.L. Ahuja. Electronic structure and magnetic properties of $\mathrm{Ni}_{2} \mathrm{MnSn}$ Heusler alloy, AIP Conference Proceedings 1349 (2011) 1123-1124.

[20] W.H. Liu, Y. Wu, J.Y. He, T.G. Nieh, Z.P. Lu. Grain growth and the Hall-Petch relationship in a high-entropy FeCrNiCoMn alloy, Scripta Mater. 68 (2013) 526-9.

[21] P.P. Bhattacharjee, G.D. Sathiaraj, M. Zaid, J.R. Gatti, C. Lee, C.W. Tsai, et al. Microstructure and texture evolution during annealing of equiatomic $\mathrm{CoCrFeMnNi}$ high-entropy alloy, J. Alloys Compd. 587 (2014) 544-52.

[22] G. Kresse, J. Hafner. Ab initio molecular dynamics for liquid metals, Phys. Rev. B 47 (1993) 558-561.

[23]G. Kresse, J. Furthmüller. Efficient iterative schemes for ab initio total-energy calculations using a plane-wave basis set, Phys. Rev. B 54 (1996) 11169-11186.

[24]L. Verlet. Computer experiments on classical fluids .i. thermodynamical properties 
of lennard-jones molecules, Phys. Rev. 159 (1967) 98.

[25]S. Nose. A unified formulation of the constant temperature molecular-dynamics methods, J. Chem. Phys. 81 (1984) 511-519.

[26]P.E. Blochl. Projector augmented-wave method, Phys. Rev. B 50 (1994) 17953.

[27]J.P. Perdew, A. Ruzsinszky, G.I. Csonka, O.A. Vydrov, G.E. Scuseria, L.A. Constantin, X.L. Zhou, K. Burke. Restoring the density-gradient expansion for exchange in solids and surfaces, Phys. Rev. Lett. 100 (2008) 136406.

[28]A. Zunger, S.H. Wei, L.G. Ferreira, J.E. Bernard. Special quasirandom structures, Phys. Rev. Lett. 65 (1990) 353-356.

[29]A. van de Walle, M. Asta, G. Ceder. The Alloy Theoretic Automated Toolkit: A user guide, CALPHAD 26 (2002) 539-553.

[30]A. van de Walle. Multicomponent multisublattice alloys, nonconfigurational entropy and other additions to the Alloy Theoretic Automated Toolkit, CALPHAD 33 (2009) 266-278.

[31]M.C. Gao, C. Niu, C. Jiang, D.L. Irving. Chapter 10: Applications of Special Quasi-Random Structures to High-Entropy Alloys. in: M.C. Gao, J.W. Yeh, P.K. Liaw, Y. Zhang, (Eds.). High-Entropy Alloys: Fundamentals and Applications. Springer, Cham, Switzerland, 2016.

[32]J.P. Perdew, K. Burke, M. Ernzerhof. Generalized gradient approximation made simple, Phys. Rev. Lett. 77 (1996) 3865-3868.

[33]H.J. Monkhorst, J.D. Pack. Special points for brillouin-zone integrations, Phys. Rev. B 13 (1976) 5188. 
[34]M. Methfessel, A.T. Paxton. Phys. Rev. B 40 (1989) 3616.

[35]H.A. Jahn, E. Teller. Stability of polyatomic molecules in degenerate electronic states. I. orbital degeneracy, Proceedings of the Royal Society of London A: Mathematical, Physical and Engineering Sciences 161 (1937) 220-235.

[36]R. Kainuma, M. Ise, C.C. Jia, H. Ohtani, K. Ishida. Phase equilibria and microstructural control in the Ni-Co-Al system, Intermetallics 4 (1996) S151-S158.

[37]R. Ducher, R. Kainuma, K. Ishida. Phase equilibria in the Ni-Co-Ga alloy system, J. Alloys Compd. 466 (2008) 208-213.

[38]R.A. Laudise, W.A. Sunder, H.M. O'Bryan, D.J. Carlson, A.F. Witt. Zochralski growth of single crystals of $\mathrm{Ni}_{3-\mathrm{x}} \mathrm{Mn}_{\mathrm{x}} \mathrm{Sn}, \mathrm{J}$. Cryst. Growth 118 (1992) 277-286.

[39]M. Gao, D. Alman. Searching for next single-phase high-entropy alloy compositions, Entropy 15 (2013) 4504.

[40]M.C. Gao, B. Zhang, S. Yang, S.M. Guo. Senary Refractory High-Entropy Alloy HfNbTaTiVZr, Metall. Mater. Trans. A (2015).

[41]L.J. Santodonato, Y. Zhang, M. Feygenson, C.M. Parish, M.C. Gao, R.J.K. Weber, J.C. Neuefeind, Z. Tang, P.K. Liaw. Deviation from high-entropy configurations in the atomic distributions of a multi-principal-element alloy, Nat Commun 6 (2015) 1-13. [42]A. Takeuchi, M.C. Gao, J.W. Qiao, M. Widom. Chapter 13 High-Entropy Metallic Glasses. in: M.C. Gao, J.W. Yeh, P.K. Liaw, Y. Zhang, (Eds.). High-Entropy Alloys: Fundamentals and Applications. Springer, Cham, Switzerland, 2016. 


\section{Figure legends}

Figure $1 \mathrm{X}$-ray diffraction patterns of the $\mathrm{CoFeMnNiX}(\mathrm{X}=\mathrm{Al}, \mathrm{Cr}, \mathrm{Ga}$, and $\mathrm{Sn})$ alloys.

Figure 2 TEM images of the as-cast CoFeMnNiX (X = Al, Ga, and Sn) alloys: (a) the bright-field image showing naonoparticles distributed uniformly in the matrix of the CoFeMnNiAl alloy; (b) the dark-field micrograph of the microstructure for the CoFeMnNiAl alloy imaged by the $\left[\begin{array}{lll}0 & 0 & 1\end{array}\right]$ superlattice reflection showing regions with different contrasts, for which the bright matrix is B2 structure and the dark nanoparticle is disordered BCC structure; (c) the corresponding diffraction pattern in the $\left[\begin{array}{lll}1 & 0 & 0\end{array}\right]$ zone axis for the CoFeMnNiAl alloy; (d) the bright-field image showing the dendritic morphology of the CoFeMnNiGa alloy; (e) the electron-diffraction pattern of the dendrite from the [011] zone axis; (f) the electron-diffraction pattern of the matrix (interdendrite) from the [011] zone axis, which presents that the matrix is a B2 structure; (g) the bright-field image showing the second particle phase distributed in the matrix for the CoFeMnNiSn alloy; (h) the electron-diffraction pattern of the matrix from the [011] zone axis, indicating that the matrix of the CoFeMnNiSn alloy is a $\mathrm{L} 2{ }_{1}$ structure; and (i) the electron-diffraction pattern of the particle from the [001] zone axis.

Figure 3 Hysteresis loops of (a) CoFeMnNiMn, (b) CoFeMnNiAl and CoFeMnNiGa, (c) CoFeMnNiSn, and (d) CoFeMnNiCr alloys at room temperature.

Figure 4 (a) Spin polarized total DOS; (b) Co $d$ partial DOS; (c) Fe $d$ partial DOS; (d) Mn $d$ partial DOS; (e) Ni $d$ partial DOS; and (f) Al $s, p$ and $\mathrm{Cr} d$ partial DOS for the FCC CoFeMnNi, FCC CoFeMnNiCr, and BCC CoFeMnNiAl from DFT calculations at zero temperature; (g) Mn d-orbital decomposed partial DOS. The vertical dotted lines indicate the Fermi level. 
Figure 5 Magnetic moments of individual atoms in (a) FCC CoFeMnNi, (b) FCC CoFeMnNiCr, and (c) BCC CoFeMnNiAl predicted from DFT calculations at zero temperature.

Figure 6 AIMD-simulated partial pair correlation functions at $\mathrm{T}=1,600{ }^{\circ} \mathrm{C}$ pertaining to Ni in (a) CoFeMnNi, (b) CoFeMnNiAl, (c) CoFeMnNiGa, and (d) CoFeMnNiSn. Noticeable preference in NiAl, NiGa, and NiSn first-nearest-neighbor pairs is observed, due to the additions of $\mathrm{Al}, \mathrm{Ga}$, and $\mathrm{Sn}$ to the base CoFeMnNi alloy, respectively. 
Table 1 Chemical compositions (in at.\%) of the CoFeMnNiX (X=Al, Cr, Ga, and Sn) alloys by SEM-EDS measurements

\begin{tabular}{|c|c|c|c|c|c|c|c|c|c|}
\hline Alloys & Area & $\mathbf{F e}$ & Co & $\mathbf{N i}$ & Mn & Al & $\mathbf{G a}$ & Sn & $\mathrm{Cr}$ \\
\hline \multirow[t]{2}{*}{ CoFeMnNi } & total & 25.9 & 25.8 & 25.5 & 22.8 & - & - & - & - \\
\hline & total & 19.7 & 19.8 & 19.6 & 18.7 & 22.2 & - & - & - \\
\hline \multirow[t]{3}{*}{ CoFeMnNiAl } & dark area & 17.6 & 19.7 & 21.6 & 15.9 & 25.2 & - & - & - \\
\hline & bright area & 22.5 & 19.7 & 17.7 & 20.9 & 19.2 & - & - & - \\
\hline & total & 19.6 & 19.4 & 19.5 & 20.2 & - & 21.3 & - & - \\
\hline \multirow[t]{3}{*}{ CoFeMnNiGa } & $\mathrm{DR}(\mathrm{FCC})$ & 27.9 & 24.5 & 17.2 & 15.8 & - & 14.6 & - & - \\
\hline & Matrix (B2) & 16.9 & 18.2 & 21.6 & 21.3 & - & 20.0 & - & - \\
\hline & total & 18.8 & 19.2 & 19.2 & 19.9 & - & - & 22.9 & - \\
\hline \multirow[t]{2}{*}{ CoFeMnNiSn } & $\operatorname{Matrix}\left(\mathrm{L} 2_{1}\right)$ & 14.6 & 15.9 & 20.2 & 16.7 & - & - & 32.5 & - \\
\hline & Precipitate (BCC) & 43.0 & 28.7 & 10.1 & 12.5 & - & - & 5.7 & - \\
\hline $\mathrm{CoFeMnNiCr}$ & total & 20.12 & 20.14 & 19.50 & 19.89 & - & - & - & 20.34 \\
\hline
\end{tabular}




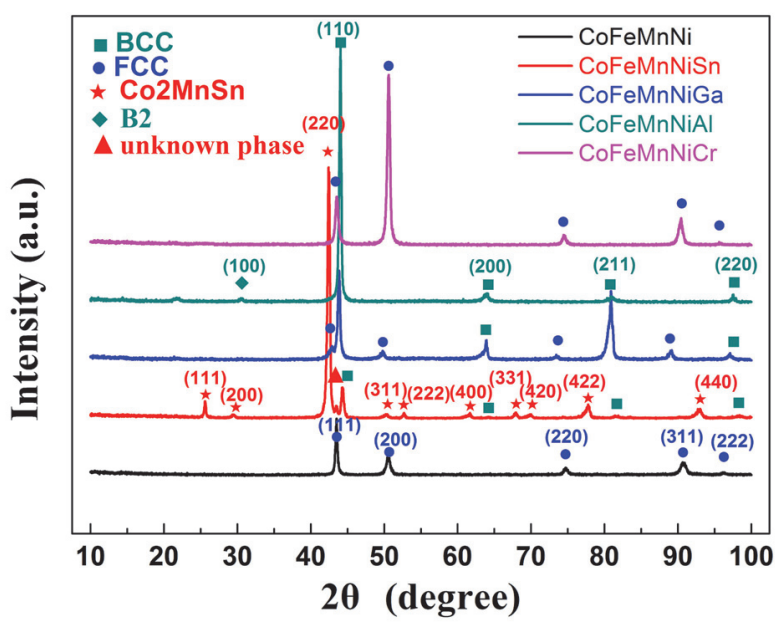




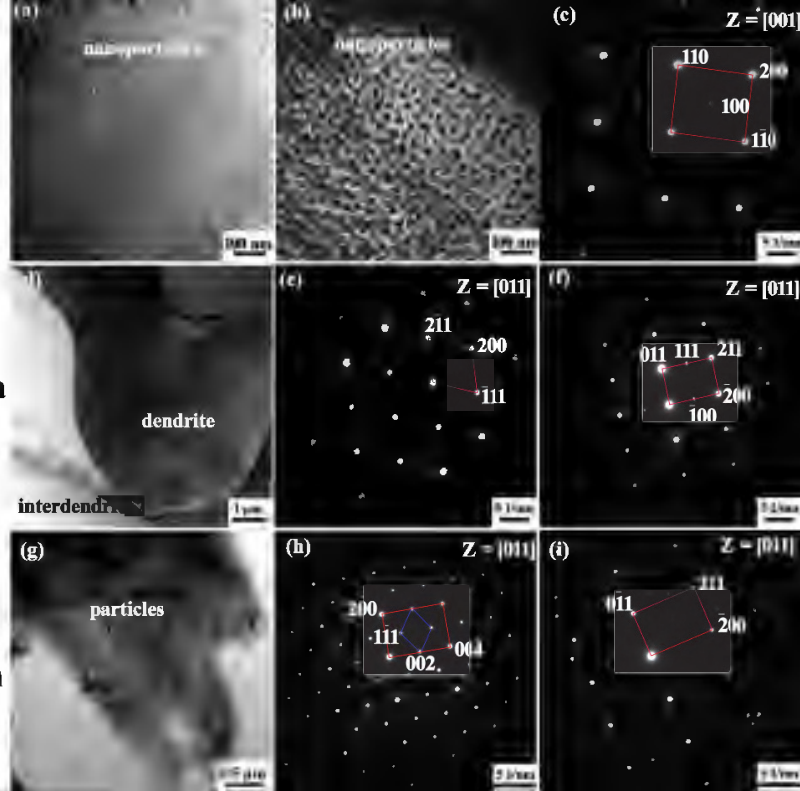



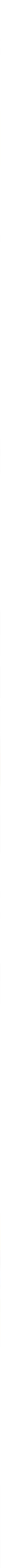


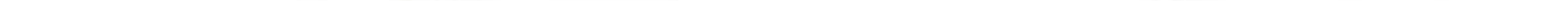




\begin{tabular}{ccc}
\hline Alloys & Ms(Am $\left.{ }^{2} / \mathrm{kg}\right)$ & $\operatorname{Hc}(\mathrm{A} / \mathrm{M})$ \\
\hline CoFeMnNi & 18.14 & $119 \mathrm{~A} / \mathrm{m}$ \\
CoFeMnNiAl & 147.86 & $629 \mathrm{~A} / \mathrm{m}$ \\
CoFeMnNiGa & 80.43 & $915 \mathrm{~A} / \mathrm{m}$ \\
CoFeMnNiSn & 80.29 & $3435 \mathrm{~A} / \mathrm{m}$ \\
CoFeMnNiCr & 1.39 & $10804 \mathrm{~A} / \mathrm{m}$ \\
\hline
\end{tabular}
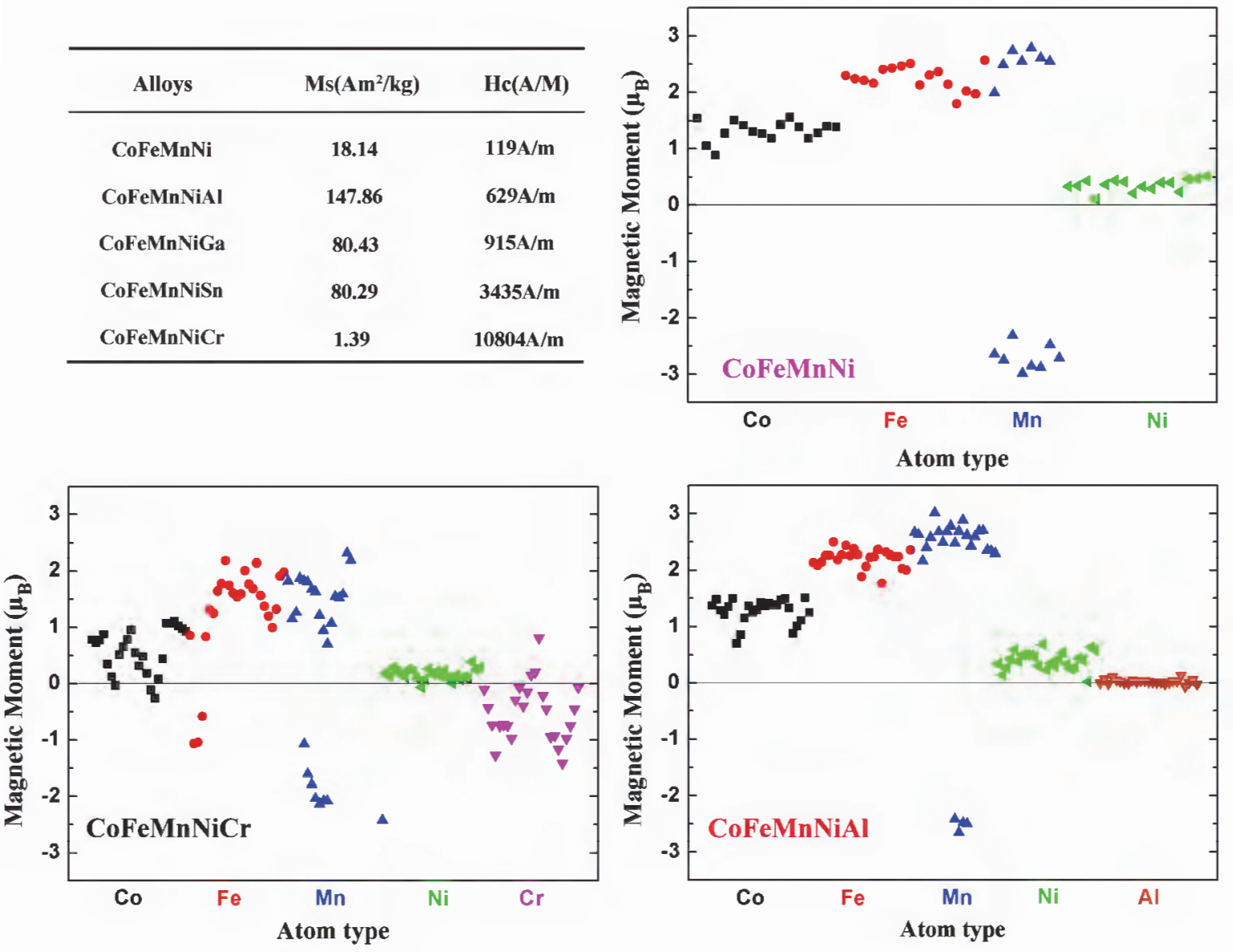